\title{
Decreased reaction time produced by discordant warning and reaction stimuli ${ }^{\prime}$
}

\author{
LAWRENCE KARLIN AND ARNOLD M. MORDKOFF
} NEW YORK UNIVERSITY

Decreased reaction time $(R T)$ was obtained when the stimulus modality of the warning signal (WS) differed from that of the reaction stimulus ( $R S)$. This effect was obtained only when the interval between the WS and $R S$ was relatively short $(.5 \mathrm{sec})$ and not when it was lengthened to $2 \mathrm{sec}$.

The present experiment was concerned with determining whether the sensory properties of a warning signal (WS) used to signal a subsequent reaction stimulus (RS) had effects additional to its cognitive significance as a signal. Specifically, we wished to determine whether the four combinations of lights and sounds (LS, SS, LL, and SL) used as warning and reaction stimuli, respectively, exercised a systematic influence on RT.

The theoretical basis for this experiment derives in part from an early controversy between Freeman and Mowrer on the role of peripheral factors in set. Mowrer and his colleagues interpreted their results to mean that peripheral factors were unimportant (Mowrer, Rayman, \& Bliss, 1940). Freeman (1940) argued that their results were not conclusive since they had not controlled for differential receptor adjustment. In support of this view he pointed to an anomalous result obtained by them. When $S$ was led to expect alternating light and sound stimuli and two sounds were unexpectedly presented in succession, RT (finger movement) to the second sound decreased. Freeman ascribed this result to proprioceptive feedback from receptor adjustments to the preceding sound stimulus which counteracted any cognitive expectation of a light stimulus (Freeman, 1940).

Whether or not Freeman's interpretation is correct, the above result suggests that RT to sound might be shorter when preceded by the same stimulus as a WS than when sound (S) is preceded by light (L), i.e., $\mathrm{RT}_{\mathrm{SS}}<\mathrm{RT}_{\mathrm{LS}}$. If these effects are general we might also expect to find $R T_{L L}<R T_{S L}$. Method

The stimuli were a brief tone of moderate intensity (S) and the flash of a neon lamp (L). Two groups, each consisting of 12 college students, were given the same conditions except that the mean foreperiod in each group was either .5 or 2 sec. Within each group foreperiods were varied $( \pm 20 \%)$ about the mean to prevent synchronization to the reaction stimulus. Intertrial intervals were approximately 10 sec. The four conditions (LS, SS, LL, SL) were presented to each $S$ in blocks of 19 trials; the blocks varied in sequence so that over the $12 \mathrm{Ss}$ in each group every condition appeared three times in each position. $S$ was informed prior to each block of the condition to follow. After a brief rest the sequence for a given $S$ was repeated in the second half of the session for a total of 152 trials which lasted about $40 \mathrm{~min}$.

\section{Results and Discussion}

The first four trials of each block served only for practice and the following analyses were based on 30 trials for each condition for each S. Table 1 presents the means for each condition for each foreperiod. Analyses of variance revealed a highly significant interaction between WS and RS only for the shorter $(.5 \mathrm{sec})$ foreperiod $(F=13.4, \mathrm{df}=1 / 33, \mathrm{p}<$ .01). With this foreperiod RT was significantly shorter when WS and RS differed.

These results do not support Freeman's hypothesis of a proprioceptive feedback mechanism which facilitates response to the second of a pair of stimuli in the same modality. Decreased RT for light followed by sound has also been reported by Hershenson (1962) but only when the interstimulus interval did not exceed about 50 or $60 \mathrm{msec}$. Since the intervals used in the present experiment were much greater and $S$ was required to respond to the second stimulus, rather than to the first as in Hershenson's experiment, the intersensory facilitation found by him seems unrelated to that obtained in the present study.

The present results might be attributed to greater arousal produced by more varied stimulation within the heteromodal blocks, but such variation does not account for the absence of differences with the longer foreperiod although an interaction with foreperiod length is possible. An alternative explanation consistent with the differential findings as a function of foreperiod length is related to stimulus generalization. Identical warning and reaction signals might lead to response conflict which would lengthen RT. Conceivably, any conflict engendered by a generalization process would be less pronounced under the longer foreperiod condition. A related issue is whether these effects can also be obtained with stimuli which differ

Table 1. Mean RT in msec by foreperiod and condition

\begin{tabular}{|c|c|c|c|c|c|c|}
\hline \multirow{2}{*}{\multicolumn{4}{|c|}{$\begin{array}{r}\text { A. } .5 \mathrm{sec} \text { foreperiod }(\mathrm{N}=12) \\
\text { Reaction } \\
\text { Stimulus } \\
\mathrm{S}\end{array}$}} & \multicolumn{3}{|c|}{$\begin{aligned} \text { B. } 2 \mathrm{sec} \text { foreperiod }(\mathrm{N}=12) \\
\text { Reaction } \\
\text { Stimulus. }\end{aligned}$} \\
\hline & & & & & $S$ & $L$ \\
\hline \multirow[b]{2}{*}{$\pi$} & & 230 & 200 & \multirow{2}{*}{ Warning Signal } & S 24 & 228 \\
\hline & $\mathrm{L}$ & 216 & 220 & & L 245 & 233 \\
\hline
\end{tabular}


within the same modality. Mowrer (1941), in a followup of his earlier study, found that RT was lengthened when the "unexpected" stimulus was in a different modality but not when it was different and in the same modality. More recently, Sutton \& Zubin (1965) have reported that $\mathrm{RT}$ on a given trial was lengthened when the stimulus on the immediately preceding trial was different. Differences between modalities were more effective than differences within modalities. Whether their findings are in any way related to ours remains to be seen since they required $S$ to make the same reaction to every stimulus (no WS was used). If our situation provided an opportunity for response conflict (preparation vs overt response) and theirs did not, stimulus generalization might either have facilitated or have had no effect in their experiments but could have interfered in ours (Osgood, 1949).

\section{References}

FREEMAN, G. L. "Central" vs. "peripheral" locus of set: A critique of the Mowrer, Rayman, and Bliss "demonstration." J. exp. Psychol., $1940,26,622-628$.

HERSHENSON, M. Reaction time as a measure of intersensory facilitation. J. exp. Psychol., 1962, 63, 289-293.

MOWRER, O. H. Preparatory set (expectancy): Further evidence of its "central" locus. J. exp. Psychol., 1941, 28, 116-133.

MOWRER, O. H., RAYMAN, N. N., \& BLISS, E. L. Preparatory set (expectancy): An experimental demonstration of its "central" locus. J. exp. Psychol., 1940, 26, 357-372.

OSGOOD, C. E. The similarity paradox in human learning. Psychol. Rev., 1949, 56, 132-143.

SUTTON, S., \& ZUBIN, J. Effect of sequence on reaction time in schizo phrenia. In A. T. Welford and J. E. Birren (Eds.), Behavior, aging, and the nervous system. Springfield, Illinois: Charles C. Thomas, 1965. P. 562-597.

\section{Note}

1. This investigation was supported in part by Public Health Service Grants MH 07253 and MH 13758. 